\title{
Extended-Spectrum Beta-Lactamase and AmpC Beta-lactamase Mediated Resistance in Escherichia coli from Clinical Sources
}

\author{
Agbagwa OE ${ }^{*}$, Aminofifori J \\ Department of Microbiology Faculty of Science University of Port Harcourt, Rivers State Nigeria, P.M.B. 5323 East-West Road Choba \\ *Corresponding author: obakpororo.agbagwa@uniport.edu.ng
}

\begin{abstract}
The development of antibiotic resistance in bacteria following the introduction of antimicrobial agents has emerged as an important medical problem worldwide. Antibiotic resistance has made the treatment of infectious diseases a global challenge. The study was carried out to detect Extended Spectrum Beta-Lactamase (ESBL) and Amp C Beta-lactamase mediated resistance of Escherichia coli. Twelve isolates were collected from the University of Port Harcourt, department of Microbiology; nine out of the 12 were identified to be E. coli after series of biochemical test. Antimicrobial susceptibility testing was carried out on all the isolates using the disc diffusion method. High level of antimicrobial resistance was observed in the test organism against Augmentin and Ampicillin. ESBL preliminary screening, ESBL phenotypic confirmatory test and double disk synergy test was performed on all the isolates to detect ESBL producers. Results obtained from the study showed that $5(55.5 \%)$ out of the nine $E$. coli isolates were confirmed to be ESBL producers. AmpC production was detected in only one of the E. coli isolate which was from wound swab. The study revealed a high rate of ESBL- producing $E$. coli from clinical specimen. Further studies should be carried out using molecular method to detect ESBL genes and a wider range of E. coli from different sources should be used.
\end{abstract}

Keywords: Amp C, Antibiotic resistance, E. coli, Extended Spectrum Beta-Lactamase

Cite This Article: Agbagwa OE, and Aminofifori J, "Extended-Spectrum Beta-Lactamase and AmpC Betalactamase Mediated Resistance in Escherichia coli from Clinical Sources." American Journal of Microbiological Research, vol. 5, no. 5 (2017): 107-112. doi: 10.12691/ajmr-5-5-3.

\section{Introduction}

Antibiotics have always been considered one of the wonder discoveries of the 20th century. The importance and value of antibiotics cannot be over emphasized; they are used to treat infectious diseases that are caused by microorganisms. Presently there is a rise of antibiotic resistance in hospitals, communities, and the environment concomitant with their use [1]. Based on the rise in antibiotic resistance infectious diseases are more difficult to treat. Recently WHO declared antibiotic resistance a top health issue worldwide, about $70 \%$ of the bacteria that cause infections are resistant to at least one of the drug most commonly used for treatment and this includes Escherichia coli which have emerged as one of the most significant causes of both nosocomial and community acquired infections [1]. $\beta$-lactams, especially extended-spectrum cephalosporin, carbapenems and flouroquinolones antibiotics represent the main therapeutic options to treat infections caused by these pathogens [2]. Extended spectrum $\beta$-lactamases (ESBLs) are class A enzyme that have the ability to hydrolyze third generation cephalosporins (ceftriaxone, cefotaxime, and ceftazidime) but are inhibited by clavulanic acid. Most of ESBLs producers are susceptible to cefoxitin and cefotetan but they are not active against cephamycins $[3,4]$.

The ability of $E$. coli to produce ESBL and AmpC have been pointed as one of the reasons for the emergence of drug resistance in Escherichia coli. The beta lactamase are enzymes that have the ability to destroy the beta lactam ring of most beta lactam antibiotics. Beta-lactamases hydrolyse small, broad or extended spectrum beta-lactam antibiotics. Extended spectrum beta-lactam (ESBL) confer resistance to most beta lactam antibiotics, including penicillins, cephalosprins, and the monobactam aztereonam leaving carbapenems as the best antimicrobial agent for infections caused by ESBL enzymes. The extensive use of antibiotics in both human medicine and for agricultural purposes, particularly in disease prevention and growth promotion in animal production is a considerable cause of the selection and prevalence of antibiotic resistant E.coli. Escherichia species are very adaptive, enteric Gram-negative rod-shaped bacterium which belongs to the family Enterobacteriaceae. They are rod shaped and do not form spores. There are six species, of which four namely: Escherichia coli, Escherichia fergusoni, Escherichia hermanii, and Escherichia vulneris are known to cause disease.

ESBL-producing Enternterobacteraceae have been responsible for numerous outbreaks of infection throughout 
the world and pose challenging infection control issues Clinical outcomes data shows that ESBLs are clinically significant and when detected they indicate the need for the use of appropriate antimicrobial agents. Unfortunately, the laboratory detection of ESBLs can be complex and at times, misleading. ESBLs and AmpC $\beta$-lactamases were first described in 1983 (Germany) and 1988 (India), respectively [5].

Antibacterial choice is often complicated by multi resistant. Many ESBL producing organisms also express AmpC beta-lactamases and may be co-transferred with plasmid mediated aminoglycoside resistance. AmpC type beta-lactamases are another group of enzymes that are commonly isolated from extended spectrum cephalosporin-resistant Gram negative bacteria. AmpC beta-lactamases are typically encoded on the chromosome of many Gram negative bacteria including Escherichia coli, Citrobacter freundii and Enterobacter spp, but can be seen on plasmids. AmpC beta-lactamases, in contrast to ESBLs, hydrolyse broad and extended spectrum cephalosporins but are not inhibited by clavulanic acid or the beta-lactamase inhibitors.

In recent years, bacteria resistance to beta-lactam antibiotics has been dramatically increased [6]. Contribution to this increase has been the spread of ESBL enzymes that hydrolyses the extended spectrum cephalosporin, like ceftazidim and cefotaxime [7].

The first AmpC beta-lactamase was detected in 1940, although at the time it was called penicillinase AmpC-producing bacteria have a broader spectrum of resistance than ESBL-producing bacteria. Next to the hydroxylation of penicillin, broad spectrum cephalosporin and extended spectrum cephalosporin of the 3rd generation cephalosporins (but not the 4th generation of cephalosporins). The synthesis of beta- lactamases is either chromosomal designated as constitutive, (as in Pseudomonas aeruginosa) or inducible plasmid mediated as in Aeromonas hydrophila, Enterobacteriaceae and Staphylococcus aureus. In the Gram positive bacteria, beta-lactamases are secreted as exo-enzymes to the outside membrane environment. However, in the Gram negative bacteria they settle in the periplasmic space and attack the antibiotics before it can reach its target site [8]. Plasmids are a major cause of spreading of bacterial resistance. This is because they can be transferred between Gram negative bacteria by conjugation and between Gram positive bacteria by bacterial viruses called transducing phages. The consequence of this transferability includes many outbreaks of resistance which are aggravated where inappropriate infection control measures are bound.

The emergence of antibiotic resistance in Escherichia coli have been attributed to its ability to produce Beta lactamsa enzymes including ESBL and AmpC Beta lactamase mediated resistance are of increasing clinical concern globally. Coudron [9] conducted a study on the occurrence and detection of AmpC beta-lactamase among E.coli, Klebsiella pneumonia and Proteus mirabilis isolates at a veterans medical center. A total of 1,286 isolates were tested 683 E.coli, $371 \mathrm{~K}$. pneumonia and 232 P. mirabilis isolates. $13(1.9 \%), 28(7.6 \%)$ and $4(1.7 \%)$, respectively, demonstrated AmpC band. Cefoxitin resistance was transferred from all but 8 (E.coli) of the $16 \mathrm{AmpC}$ producers. The present study was carried out to detect
ESBL and AmpC Beta-lactamase mediated resistance in Escherichia coli isolates of clinical origin.

\section{Materials and Methods}

\subsection{Samples Collection and isolation of E.coli}

A total of 12 isolates were collected from the Microbiology laboratory of the University of Port Harcourt. All isolates were sub cultured on Eosin methylene blue Agar according to manufacturer's specification; the plates were incubated at $37^{\circ} \mathrm{C}$ for 24 hours. After which biochemical tests were carried out in order to identify Escherichia coli strain.

\subsubsection{Identification of Escherichia coli Characterization and Identification of Escherichia coli}

The bacteria isolates were grown on Eosin Metylene Blue Agar (LAB M), they were sub cultured onto Nutrient Agar (LAB M). E. coli was characterized and identified based on their motility, microscopic morphology, colonial morphology and biochemical characterization as described in medical laboratory manual for tropic countries Cheesbrough and with reference to the Bergey's manual of systemic bacteriology $[10,11]$.

\subsection{Antibiotic Susceptibility test}

Susceptibility was determined by the Kirby Bauer disc diffusion method as described by National Committee for Clinical and Laboratory Standard [12]. Bacteria were grown on nutrient broth at $37^{\circ} \mathrm{C}$ overnight. The suspension was visually adjusted with normal saline to equal that of 0.5 Mac Farland turbidity standards. The inoculum was swabbed across the entire surface of Mueller Hinton agar plate (Biotech) using sterile swab stick and the plate was rotated approximately $60^{\circ} \mathrm{C}$ between streaking to ensure an even distribution. Inoculated plates were left to stand for at least 3 minutes, but for no longer than 15 minutes before the disks were applied. Commercial antibiotics disks used include: Ceftazidime (30ug), Cefuroxime (30ug), Gentamicin (10ug), Ciprofloxacin (5ug), Ofloxacin (5ug), Amoxicillin/Clavulanate (30ug), Nitrofurantoin (300ug), Ampicillin (10ug), (TOKU-E, USA). The plates were incubated within 15 minutes of the application of the disks at $37^{\circ} \mathrm{C}$ for 18 to $24 \mathrm{~h}$. The inhibition zone diameters around the disks were measured and interpreted according into the NCCLS guidelines [12]. Isolate were considered as multidrug resistance (MDR), when it showed resistance to $\geq 3$ antimicrobial agents [13].

\subsection{Detection of Extended Spectrum Beta Lactamase}

For the detection of ESBL, three methods: CLSI screening method, CLSI phenotypic confirmatory method and double disk diffusion synergistic method were used.

\subsubsection{Screening test for ESBL}

Ceftazidime, ceftriaxone and cefotaxime discs were placed on a Mueller Hinton Agar plate at appropriate distance. The plates were incubated aerobically overnight 
(18-24 hours $\left./ 35^{\circ} \mathrm{C}\right)$. Isolates showing an inhibition zone size of $\leq 22 \mathrm{~mm}$ with ceftazidime $(30 \mu \mathrm{g}), \leq 25 \mathrm{~mm}$ with cefriaxone $(30 \mu \mathrm{g})$ and $\leq 27 \mathrm{~mm}$ with cefotaxime $(30 \mu \mathrm{g})$ were identified as potential ESBL producers and were short listed for confirmation of ESBL production [14].

\subsubsection{Phenotypic Confirmatory Test for ESBL}

Strains that were suspected to be ESBL producer by screening method were confirmed for enzyme production by phenotypic confirmatory disk diffssusion method. Ceftazidime disk without clavulanic acid and ceftazidime with clavulanic acid combination disk were placed on the same plate. The plates with disks were incubated aerobically overnight $\left(18-24\right.$ hours $\left./ 35^{\circ} \mathrm{C}\right)$. The isolates showing an increase in zone size of $5 \mathrm{~mm}$ or more around ceftazidime with clavulanic acid as compared to ceftazidime alone were confirmed to be ESBL producer. No enhancement of zone indicates ESBL non-producer isolates [14].

\subsubsection{The Double Disc Synergy Test (DDST)}

The double disc synergy test (DDST) method described by CLSI (2013) was employed. Standardized inoculum of the test organisms was inoculated on Mueller Hinton Agar (MHA) (BIOTECH, England) using sterile swab sticks. Amoxicillin/clavulanic acid disc $(20 / 10 \mu \mathrm{g}$, TOKU-E, USA) was placed at the center of the inoculated MHA. Ceftazidime (30ug, TOKU-E, USA) and Cefotaxime (30ug, TOKU-E, USA) were placed $15 \mathrm{~mm}$ center to center from the Amoxycillin/clavulanic acid discs. The plates were incubated at $37^{\circ} \mathrm{C}$ for 24 hours. After incubation, enhancement of zone of inhibition of either or both the Ceftazidime and Cefotaxime discs towards the Amoxycillin/Clavulanic acid discs is indicative of ESBL production.

\subsection{Detection of AmpC Beta-lactamase Production}

\subsubsection{Disk Diffusion Method}

The Double Disk Synergy test was done using the CLSI 2013 method. Mueller Hilton Agar plate were inoculated with the suspension of the standard inoculum of the test organism, it was left for 15 minutes before disc containing 30 ug cefoxitin and 400ug of Boronic acid were placed on each of the inoculated plate $20 \mathrm{~mm}-25 \mathrm{~mm}$ apart. The plates were inoculated at $37^{\circ} \mathrm{C}$ for 18 hours. The organism that demonstrated $5 \mathrm{~mm}$ or greater $5 \mathrm{~mm}$ zone around the disk containing cefoxitin and boronic acid compared to the disk containing cefoxitin was considered as AmpC producer.

\section{Results and Discussion}

3.1. Twelve isolates were obtained from the University of Port Harcourt Teaching hospital. Nine of the isolates were identified as Escherichia coli after series of biochemical test which include dindole, citrate, motility, TSIA, Methyl Red test and Voges Proskauer test. Table 1 shows the detailed result of biochemical test for the nine isolates.
Table 1. Biochemical Test Result

\begin{tabular}{|c|c|c|c|c|c|c|c|c|c|}
\hline \multirow{2}{*}{ Isolates } & \multirow{2}{*}{ Indole } & \multirow{2}{*}{ MR } & \multirow{2}{*}{$\mathbf{V P}$} & \multirow{2}{*}{ Citrate } & \multicolumn{4}{|c|}{ TSIA } & \multirow{2}{*}{ Organism } \\
\hline & & & & & Slant & Butt & $\mathrm{CO}_{2}$ & $\mathrm{H}_{2} \mathrm{~S}$ & \\
\hline Iso 1 & + & - & - & + & $\mathrm{K}$ & $\mathrm{K}$ & + & - & E. coli \\
\hline Iso 2 & + & - & - & + & $\mathrm{K}$ & $\mathrm{K}$ & + & - & E. coli \\
\hline Iso 3 & - & + & - & - & A & A & + & - & S. aureus \\
\hline Iso 4 & + & - & - & + & $\mathrm{K}$ & $\mathrm{K}$ & + & - & E. coli \\
\hline Iso 5 & + & - & - & + & $\mathrm{K}$ & $\mathrm{K}$ & + & - & E. coli \\
\hline Iso 6 & + & - & - & + & $\mathrm{K}$ & $\mathrm{K}$ & + & - & E. coli \\
\hline Iso 7 & - & + & - & - & A & A & + & - & S. aureus \\
\hline Iso 8 & + & - & - & + & $\mathrm{K}$ & $\mathrm{K}$ & + & - & E. coli \\
\hline Iso 9 & - & + & - & + & A & A & + & - & S. aureus \\
\hline Iso 10 & + & - & - & + & $\mathrm{K}$ & $\mathrm{K}$ & + & - & E. coli \\
\hline Iso 11 & + & - & - & + & $\mathrm{K}$ & $\mathrm{K}$ & + & - & E. coli \\
\hline Iso 12 & + & - & - & + & $\mathrm{K}$ & $\mathrm{K}$ & + & - & E. coli \\
\hline
\end{tabular}

$\mathrm{KEY}: \mathrm{A}=$ acidic condition, $\mathrm{K}=$ alkaline condition, $+=$ positive, - = negative.

\subsection{Distribution of Antibiotic Resistance among $\boldsymbol{E}$. coli Isolates}

All isolates were examined for resistance to six antibiotics. Varying degree of resistance was noticed with the various groups of antimicrobial agents used as shown in Figure 1. All isolates showed susceptibility to Ceftazidime (30ug), Cefuroxime (30ug), Gentamicin (10ug), Ciprofloxacin (5ug), Ofloxacin (5ug), Amoxicillin/Clavulanate (30ug), Nitrofurantoin (300ug), Ampicillin (10ug). High resistance was found in Amoxicillin/clavulanate and Ampicillin. Percentage susceptibility result is shown in Figure 1.

\subsection{Prevalence of Extended Spectrum Beta-lactamase and AmpC among Escherichia coli Isolates}

Extended spectrum and AmpC beta-lactamase was detected in during analysis of isolates. Among the isolates $7(77.8 \%)$ was positive for ESBL and 1(11.1\%) was positive for AmpC as indicated in Table 2. Prevalence was shown in ESBL more than AmpC (Figure 2).

Table 2. Distribution of Extended spectrum beta-lactamase and AmpC among Escherichia coli isolates

\begin{tabular}{ccccc}
\hline Isolates & $\begin{array}{c}\text { ESBL } \\
\text { screening }\end{array}$ & $\begin{array}{c}\text { Phenotypic } \\
\text { confirmatory test }\end{array}$ & DDST & AmpC \\
\hline Iso1 & + & - & - & - \\
Iso2 & + & + & + & - \\
Iso4 & + & - & - & - \\
Iso5 & + & + & + & - \\
Iso6 & + & + & + & + \\
Iso8 & + & + & + & - \\
Iso10 & + & + & + & - \\
Iso11 & + & - & - & - \\
Iso12 & + & - & - & - \\
\hline
\end{tabular}

KEY : + = Positive, $-=$ Negative 


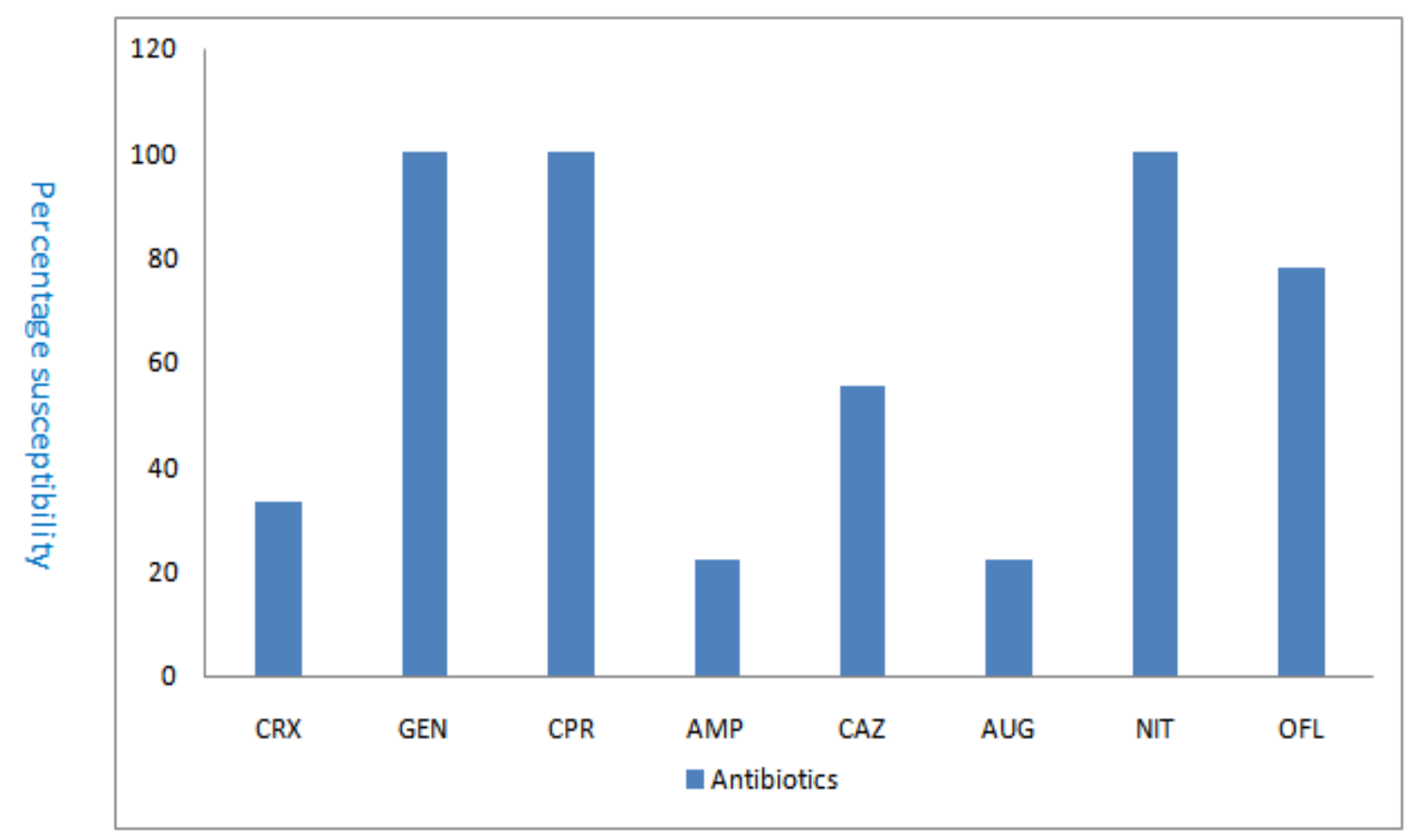

Figure 1. Percentage susceptibility of Escherichia coli isolates against test antibiotics

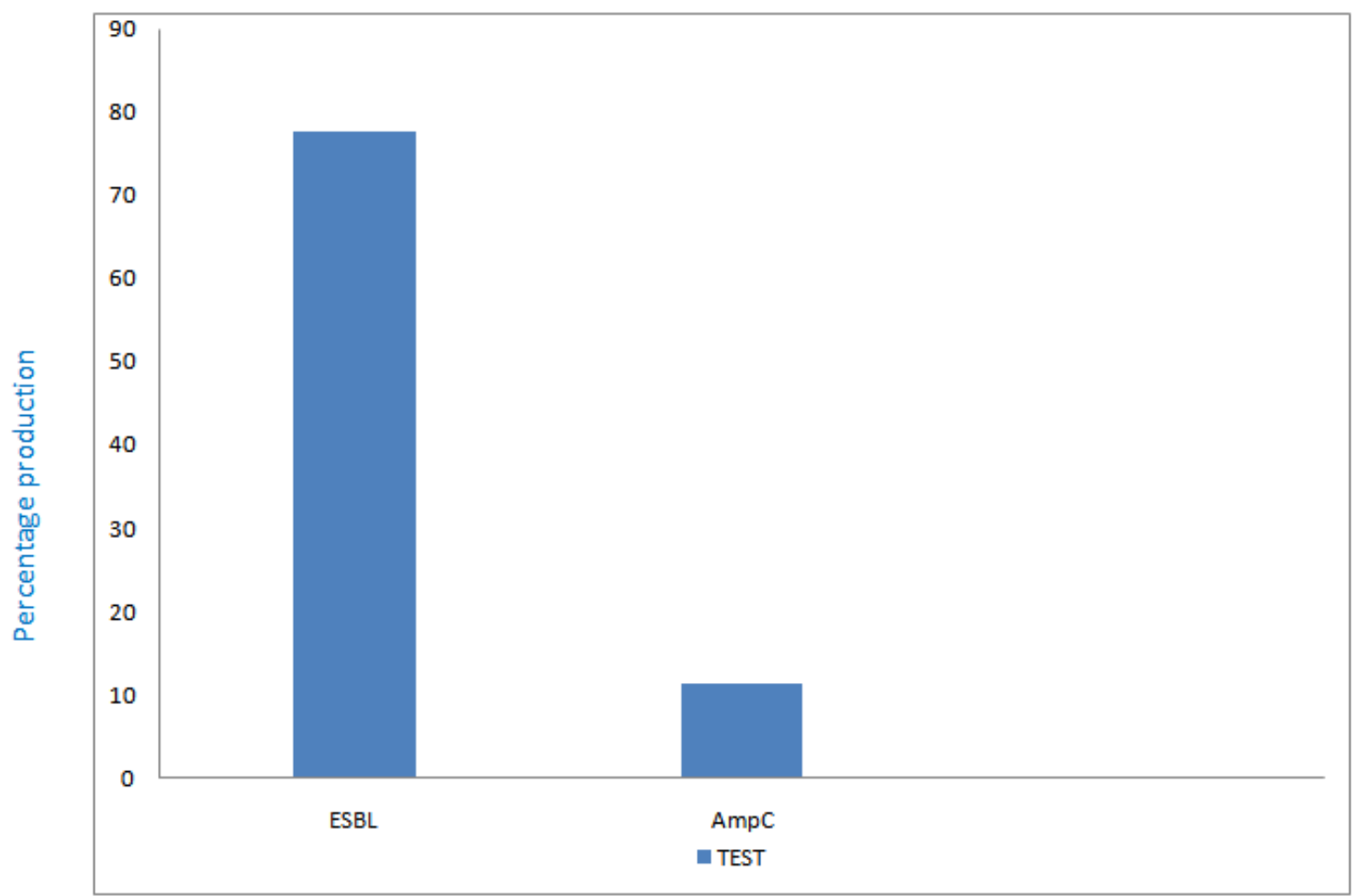

Figure 2. Percentage Production of ESBL and AmpC among Escherichia coli isolates
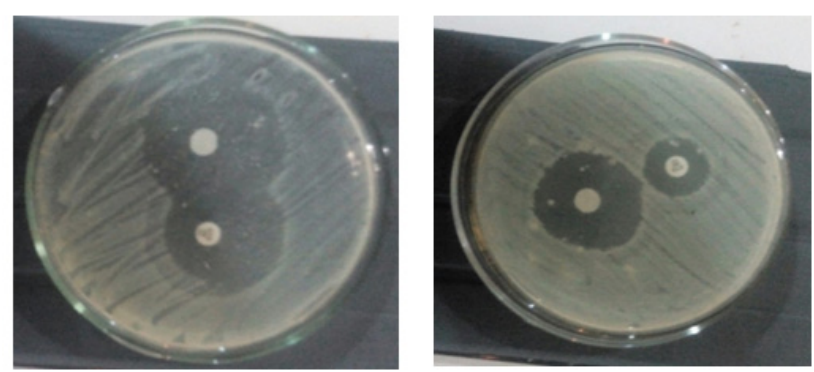

Plate 1. Comparison of Beta-lactamase production of Escherichia coli isolates
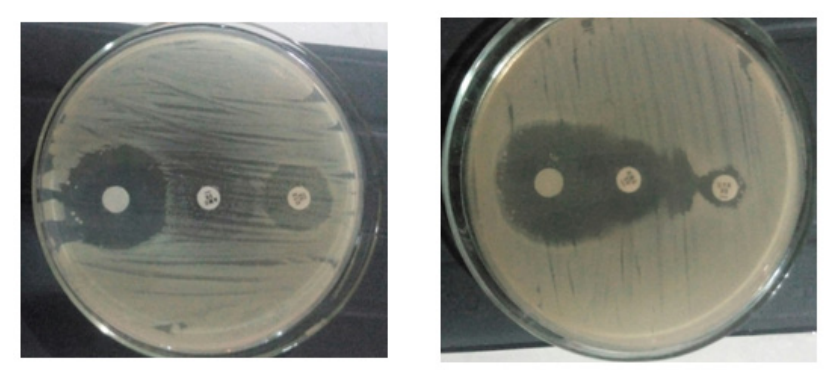

Plate 2. Positive and negative double disc synergy test 

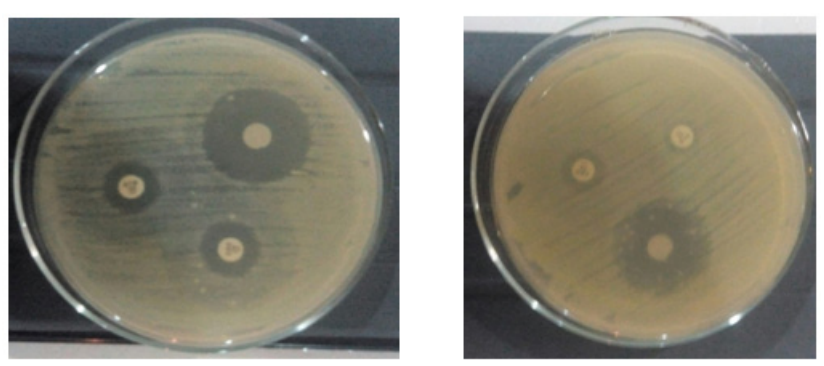

Plate 3. Positive and negative AmpC disc diffusion test of Escherichia coli isolate

The emergence of multi-drug resistant bacterial strains in hospitals where antibiotic use is frequent and the patients are in critical condition is a growing concern [15]. Several studies have been undertaken to assess the susceptibility of bacterial pathogens to different antibiotics. An in vitro surveillance study was conducted in Saudi Arabia to assess antibiotic susceptibility patterns among $E$. coli isolates. Inpatients isolates were more likely to be antibiotic resistant than outpatient isolates, as observed in the resistance patterns to ampicillin (63\% in inpatients, $50 \%$ in outpatients), sulfamethoxazol (44\% in inpatients, $30 \%$ in outpatients), and ciprofloxacin (33\% in inpatients, $14 \%$ in outpatients). These findings revealed that there is wide spread resistance to most of the available antibiotics [16]. Also susceptibility study conducted in California University on prospectively collected $255 \mathrm{E}$. coli isolates showed $22 \%$ resistance to trimetoprim-sulfamethoxazole.

ESBLs are clinically important because they destroy cephalosporins, given as first line agents to many severely ill patients [17]. Therapeutic options for the infections which are caused by the ESBL producers have also become increasingly limited [18]. Broad spectrum resistance raises concern and necessitates the restricted use of extendedspectrum cephalosporin, and a trial of other suitable alternatives [19].

In this study, $77.8 \%$ of the $E$. coli isolates were positive for ESBL screening using cefotaxime, ceftazidime and ceftriaxone $30 \mu \mathrm{g}$ each discs as initial screening agent which were further subjected to confirmatory tests. Factors that can interrupt test result include disk placement, correct storage of the clavulanic discs and performance of appropriate control tests as they are critical to the sensitivity of DDST. Double disk test can lack sensitivity because of the problems of optimal discs spacing and the correct storage of the clavulanic acid containing discs [20,21] conducted a study at a 900+ bed general hospital, from May to September 2007, in Iran, to determine the prevalence of ESBL producing E.coli and K. pneumonia and their antimicrobial pattern. Results obtained from their study showed $100 \mathrm{~K}$. pneumonia and 106 E.coli isolates were susceptible to imipenem. From the Female isolates (136), 59.5\% and (70) 58.6\% were ESBL producing group.

High-level AmpC production is typically associated with in-vitro resistance to third-generation cephalosporins and cephamycins. In connection with this, high clinical treatment failures with broad-spectrum cephalosporins have been documented [22]. In this study AmpC $\beta$-lactamase production was confirmed in $1(11.1 \%)$ of 9 isolates and in the remaining 8 it was not detectable and this correspond with the result of Shayan and Bokaeian in their study of Detection of ESBL- and AmpC-producing E. coli isolates from urinary tract infections in Iraq [23].

\section{Conclusion}

ESBLs and AmpC $\beta$-lactamases enzymes have been of increasing importance based on their ability to mediate resistance in most bacteria. There is a need for a correct and reliable phenotypic test to identify AmpC $\beta$ lactamases and to discriminate between AmpC and ESBL producers. Also, the ESBLs and AmpC production monitoring is recommended to avoid treatment failure and for suitable infection control. Some ESBLs may reach a level to be detectable by disk diffusion tests which could result in treatment failure in the infected patient.

\section{References}

[1] Rajiv, D. Disease, illness, education, public awareness, science and technologyand antimicrobial resistance. 2016.

[2] Cantón R., Novais A., Valverde A., Machado E., Peixe L., Baquero, F. Prevalence and spread of extended-spectrum betalactamase-producing Enterobacteriaceae in Europe. Clin Microbiol Infect, 2008, 1:44-53.

[3] Martine'z-Martine'z, L., Herna'ndez-Alle's, S., Albertı's, S. Toma's J., Benedi, V. In vivo selection of porin-deficient mutants of Klebsiella pneumoniae with increased resistance to cefoxitin and expanded-spectrum-cephalosporins. Antimicrob. Agents Chemother, 1996, 40, 342-348.

[4] Bradford, PA. Extended - spectrum beta-lactamases in the $21^{\text {st }}$ century: characterization, epidemiology, and detection of this important resistance threat. Clin Microbiol Rev, 2001, 14, 933-951.

[5] Thomson, KS. Controversies about extended-spectrum and AmpC beta-lactamases. Emerg Infect Dis, 2001, 7, 3-6.

[6] Kalayama, Y., Zhang, HZ., Chambers, HF. PBP mutations producing very-high- level resistance in beta-lactamase. Antimicrobial Agents Chem. 2004, 48, 453-459.

[7] Davies, TA., Shang W., Bush K., Flamm, RK. Activity of Imepenem and comparator beta-lactam agent clinical isolates of streptococcus pneumonia with defined motivations in the penicillin binding domain of pbp2b and pbp2x. Journal of A.M chemother. 2008, 6(3); 751-753.

[8] Stratton, CW. Mechanisms of bacterial resistance to antimicrobial agents. 2000.

[9] Coudron, PE. Inhibitor-based methods for detection of plasmidmediated AmpC beta-lactamases in Klebsiella spp., Escherichia coli, and Proteus mirabilis. Journal of Clinical Microbiology, 2005, 43:416-437.

[10] Cheesbrough M. District laboratory practice in tropical countries, part 2. Cambridge University Press, Cambridge, 2005, 159-162.

[11] Krieg, NR and Holt, JW. Bergey's Manual of bacteriology, $9^{\text {th }}$ edition, Williams and Wilkins, Baltimore. 1994.

[12] CLSI. Performance Standards for Antimicrobial Susceptibility Testing. In: Twenty-third Informational Supplement. CLSI Document M100-S23, Pennsylvania. Clinical and Laboratory Standards Institute, 2013, 34-190.

[13] Gayathri, G.,Kathireshan, AK., Balagurunathan, R. Prevalence of extended spectrum Beta Lactamases in Uropathogenic E. coli and Klebsiella species in a Chennai Suburban Tertiary Care Hospital and its Antibiogram Pattern. Research Journal of Microbiology, 2011, 6: 796-804.

[14] Giriyapur, RS., Nandihal, NW., Krishna, BVS., Patil, AB., Chandrasekhar, MR. Comparison of Disc Diffusion Methods for the Detection of Extended-Spectrum Beta Lactamase-Producing Enterobacteriaceae. Journal of Laboratory Physician, 2011, 3: 33-36.

[15] Shahanara, B., Abdus Salam, MD., Faisal Alam, KH., Nurjaham, B., Harsan, JA. Detection of extended spectrum beta- lactamase in Pseudomonas spp isolated from two tertiary hospitals in Bangladesh. 2013, BMC Research Notes, 6, 1-6. 
[16] Altayar. MA., Thokar. MA., Mohammad. MA. Extended spectrum beta-lactamase- producing Escherichia coli in clinical isolates in benghazi, libya: phenotypic detection and antimicrobial susceptibility pattern. Medical Journal of Islamic World Academy of Sciences 2012, 20:49-56.

[17] Ahmed BO, Omar AO, Asghar AH, Elhassan MM. Increasing Prevalence of ESBL- Producing Enterobacteriaceae in Sudan Community Patients with UTIs. Egyptian Academic Journal of Biological Science. 2013; 5: 17-24.

[18] Metri, BC., Jyothi, P., Peerapur, V. The prevalence of ESBL among enterobacteriaceae in tertiary care hospital of North Karnataka India. J.Clin. Diagn. Res, 2011, 5:470-475.

[19] Amita, J., Indranil, R., Mahenda, KG., Mala, K., Agarwal, SK. Prevalence of extended spectrum betalactamase producing Gram negative bacteriamin septicaemic neonates in a tertiary care hospital. Journal of medical microbiology, 2003, 52: 421-425.

[20] Wadekar, MD., Anuradha K., Venkatesha D. Phenotypic detection of ESBL and MBL in clinical isolates of Enterobacteriaceae. Into.J.Current.Red.Aca.Rev; 2013, 1: 89-95.

[21] Bazzaz, BS., Naderinasab, M., Mohamadpoor, AH., Farshahzadeh, Z., Ahmadi, S., Yousefi, F. The prevalence of extended spectrum Beta-lactamase producing Escherichia coli and Klebsiella pneumonia among clinical isolates from a general hospital in Iran. Acta Microbiologica et Immunologica Hungarica, 2009, 56: 89-99.

[22] Doi, Y. and Paterson, DL. Detection of plasmid-mediated class C beta-lactamases. Int J Infect Dis, 2007, 11: 191-7.

[23] Shayan, S. and Bokaeian, M. Detection of ESBL - and AmpCproducing $E$. coli isolates from urinary tract infections. Advanced biomedical research, 2015, 4: 220 -223. 